\title{
Mesure du coefficient d'absorption et de la transmission dérivée dans $\mathbf{G a}_{1-x} \mathbf{A l}_{x} \mathbf{A s}$ epitaxial
}

\author{
R. Madelon \\ Laboratoire de Physique des Solides (*) de l'Université de Caen, 14032 Caen Cedex, France
}

(Reçu le 13 mars 1979, révisé le 20 juin 1979, accepté le 27 juin 1979)

\begin{abstract}
Résumé. - Une méthode simple de polissage mécanique et chimique permet d'éliminer le substrat de couches minces de $\mathrm{Ga}_{1-x} \mathrm{Al}_{x} \mathrm{As}$, de quelques dizaines de $\mu \mathrm{m}$ d'épaisseur, obtenues par croissance épitaxiale sur GaAs. La mesure du coefficient de réflexion et de transmission permet de calculer le coefficient d'absorption, la largeur de la bande interdite et le paramètre d'élargissement dû aux collisions, au voisinage de la transition fondamentale $\Gamma_{15}^{\mathrm{v}} \rightarrow \Gamma_{1}^{\mathrm{c}}$. La dérivée de la transmission obtenue par modulation de longueur d'onde offre une deuxième méthode de mesure de l'énergie et de la largeur de la transition, en bon accord avec la première.
\end{abstract}

\begin{abstract}
The GaAs substrate of LPE grown, a few $\mu \mathrm{m}$ thick $\mathrm{Ga}_{1-x} \mathrm{Al}_{x} \mathrm{As}$ epitaxial layers is eliminated by simple mechanical and chemical etching. Transmission and reflectivity measurements yield the absorption coefficient, the bandwith and the collisional broadening in the vicinity of the $\Gamma_{15}^{\mathrm{v}} \rightarrow \Gamma_{1}^{\mathrm{c}}$ fundamental transition. The transmission derivative, as obtained by wavelength modulation spectroscopy, leads to values of the energy and the linewidth of the transition in good agreement with the previous one.
\end{abstract}

1. Introduction. - L'étude spectroscopique des composés pseudo-binaires s'est beaucoup développée au cours de ces dernières années en raison de l'intérêt technique de ces matériaux. Le composé $\mathrm{Ga}_{1-x} \mathrm{Al}_{x} \mathrm{As}$ a reçu une attention toute particulière car il entre dans l'élaboration de nombreux dispositifs à hétérostructures pour guides optiques, sources laser, sources électroluminescentes, etc... La position en énergie des principales transitions en fonction de la composition est assez bien connue [1-4]. Dans la plupart des cas, les mesures de spectroscopie sont effectuées par photoluminescence $[2,3,5,6]$, photoréponse [1], réflectivité ou réflectivité modulée $[4,7,8]$, car ce matériau n'existe que sous forme de couche épitaxiale. Aussi existe-t-il très peu d'études en transmission, au voisinage de la transition fondamentale $\Gamma_{15}^{\mathrm{v}} \rightarrow \Gamma_{1}^{\mathrm{c}}$. Outre qu'elle permet une mesure du coefficient d'absorption dont la connaissance est essentielle dans les applications, la transmission présente une bien meilleure sensibilité et est beaucoup plus à l'abri des imperfections de l'interface échantillon-substrat. Elle nécessite, en contrepartie, de résoudre préalablement le délicat problème de l'élimination du substrat. Zembatov et al. [9] ont utilisé un substrat transparent en GaP, Monemar, Shih et Petet [6], Sommers et

(*) Associé au C.N.R.S. n” 251.
Lockwoord [10] ont éliminé le substrat par méthode chimique sélective.

Nous présentons ci-dessous les résultats de mesures effectuées en transmission, réflexion, et transmission modulée sur des couches épitaxiales dont le substrat a été éliminé par des moyens mécaniques et chimiques sans trop de dommage pour les couches.

2. Préparation des échantillons. - Les échantillons que nous avons étudiés ont été obtenus par épitaxie en phase liquide, sur substrat de GaAs, au centre de Caen de la Radiotechnique-Compelec. Les épaisseurs de ces couches sont de l'ordre de quelques dizaines de $\mu \mathrm{m}$.

La nature du dopant et le taux de dopage, déterminés à l'aide du minimum de réflectivité infrarouge, sont donnés tableau I. Ces mesures ont été faites à la température ambiante. Les différents paramètres nécessaires, fréquence des phonons optiques longitudinaux et transversaux, permittivités, masses effectives, ont été calculés par interpolation linéaire entre ceux de GaAs $[10,11]$ et ceux de AlAs $[12,13]$.

Plutôt que d'utiliser la méthode chimique sélective décrite par Logan et Reinhart [14], nous avons éliminé le substrat par une méthode de polissage mécanique suivie d'un polissage chimique. Cette 
Tableau I. - Energie $E_{0}+\delta E$ de la transition fondamentale $\Gamma_{15}^{\mathrm{v}}-\Gamma_{1}^{\mathrm{c}}$ et largeur de raie $\Gamma$ mesurée à partir de l'absorption et de la transmission dérivée dans les échantillons étudiés.

[Energy $E_{0}+\delta E$ and line width $\Gamma$ of the fundamental transition $\Gamma_{15}^{\mathrm{v}}-\Gamma_{1}^{\mathrm{c}}$ measured by absorption and derivative transmission on three samples.]

\begin{tabular}{|c|c|c|c|c|c|c|}
\hline Echantillons & $x$ & Dopant & $\begin{aligned}- & E_{15}^{\mathrm{v}}+E_{1}^{\mathrm{c}}+\delta E \\
& \text { (absorption) }\end{aligned}$ & $\begin{array}{c}-E_{15}^{\mathrm{v}}+E_{1}^{\mathrm{c}}+\delta E \\
\text { (transmission } \\
\quad \text { dérivée) }\end{array}$ & $\begin{array}{c}\Gamma \\
\text { (absorption) }\end{array}$ & $\begin{array}{c}\Gamma \\
\text { (transmission } \\
\text { dérivée) }\end{array}$ \\
\hline$\overline{\mathrm{GaAs}}$ & $\overline{0}$ & $n=5 \times \overline{10^{15} / \mathrm{cm}^{3}}$ & - & $\overline{1404} \mathrm{eV}$ & $465 \times-0^{12} \mathrm{~s}^{-1}$ & $4,30 \times \overline{10^{12} \mathrm{~s}^{-1}}$ \\
\hline $\begin{array}{c}451 \\
(300 \mathrm{~K})\end{array}$ & $22 \%$ & $\begin{array}{c}\text { dopé } \mathrm{Ge} \\
p=1,4 \times 10^{18} / \mathrm{cm}^{3}\end{array}$ & $1,698 \mathrm{eV}$ & $1,705 \mathrm{eV}$ & $1,01 \times 10^{13} \mathrm{~s}^{-1}$ & $1,11 \times 10^{13} \mathrm{~s}^{-1}$ \\
\hline $\begin{array}{c}1 \\
(300 \mathrm{~K})\end{array}$ & $21 \%$ & $\begin{array}{c}\text { dopé } \mathrm{Ge} \\
p=1,4 \times 10^{18} / \mathrm{cm}^{3}\end{array}$ & $1,684 \mathrm{eV}$ & $1,684 \mathrm{eV}$ & $1,75 \times 10^{13} \mathrm{~s}^{-1}$ & $1,91 \times 10^{13} \mathrm{~s}^{-1}$ \\
\hline $\begin{array}{c}929 \\
(300 \mathrm{~K})\end{array}$ & $46 \%$ & $\begin{array}{c}\text { dopé Sn } \\
n=9,2 \times 10^{17} / \mathrm{cm}^{3}\end{array}$ & $2,018 \mathrm{eV}$ & $2,030 \mathrm{eV}$ & $1,06 \times 10^{13} \mathrm{~s}^{-1}$ & $1,14 \times 10^{13} \mathrm{~s}^{-1}$ \\
\hline $\begin{array}{c}451 \\
(77 \mathrm{~K})\end{array}$ & & & $1,777 \mathrm{eV}$ & $1,789 \mathrm{eV}$ & $7,9 \times 10^{12} \mathrm{~s}^{-1}$ & $6,68 \times 10^{12} \mathrm{~s}^{-1}$ \\
\hline $\begin{array}{c}1 \\
(77 \mathrm{~K})\end{array}$ & & & $1,747 \mathrm{eV}$ & $1,751 \mathrm{eV}$ & $1,42 \times 10^{13} \mathrm{~s}^{-1}$ & $1,41 \times 10^{13} \mathrm{~s}^{-1}$ \\
\hline $\begin{array}{c}929 \\
(77 \mathrm{~K})\end{array}$ & & & $2,115 \mathrm{eV}$ & $2,123 \mathrm{eV}$ & $7,56 \times 10^{12} \mathrm{~s}^{-1}$ & $7,87 \times 10^{12} \mathrm{~s}^{-1}$ \\
\hline
\end{tabular}

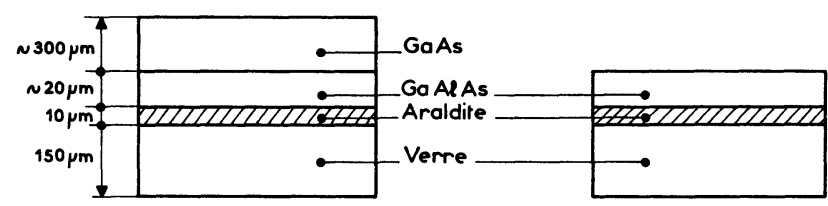

Fig. 1. - a) La couche épitaxiale GaAlAs/GaAs est initialement collée sur une plaque de verre ; $b$ ) Le substrat est éliminé mécaniquement puis chimiquement.

[a) The epitaxial layer GaAlAs/GaAs is initialy sticked on a slab of glass ; $b$ ) The substrate is eliminated by mecanical and chemical etching.]

méthode a l'avantage de conduire à des surfaces utiles nettement plus grandes.

L'échantillon qui a subi un premier polissage chimique d'une durée d'environ $10 \mathrm{~s}$ dans un mélange chaud de $1 \mathrm{H}_{2} \mathrm{O}, 3 \mathrm{H}_{2} \mathrm{SO}_{4}, 1 \mathrm{H}_{2} \mathrm{O}_{2}$ (vitesse d'attaque $10 \mu \mathrm{m} / \mathrm{min}$. environ) est collé avec de la colle époxy Araldite sur une plaque de verre de $150 \mu \mathrm{m}$ d'épaisseur (Fig. 4). L'épaisseur de colle est d'environ $10 \mu \mathrm{m}$. Ce premier traitement a pour but de parfaire les qualités optiques de la face qui va être collée. La plus grande partie du substrat est éliminée par un premier polissage mécanique à l'aide de disques abrasifs LAMPLAN série 500. L'épaisseur restante d'échantillon est contrôlée périodiquement pendant cette opération. Un polissage à la pâte diamant de grains de grosseurs dégressives $(6 \mu \mathrm{m}, 3 \mu \mathrm{m}$ et $1 \mu \mathrm{m})$ et un polissage chimique dans le mélange précédemment cité permettent d'obtenir un poli optique.

Pour s'assurer que le substrat a été complètement éliminé les échantillons non transparents à l'œil $(x<0,1)$ sont soumis à une mesure de transmission. Le polissage chimique est alors recommencé le cas échéant.
La couche épitaxiale ainsi obtenue a une épaisseur de 10 à $30 \mu \mathrm{m}$ et une surface utile de $0,5 \mathrm{~cm}^{2}$.

On vérifie que dans la bande spectrale de travail $(1,2 \mathrm{à} 2,5 \mathrm{eV})$ le verre et l'araldite n'apportent aucune modification notable au spectre. L'atténuation produite est très faible et peut d'ailleurs être compensée par le dispositif expérimental.

3. Dispositif expérimental et mesures. - Les mesures ont été effectuées sur un spectromètre modulaire Coderg permettant de mesurer la réflexion, la transmission et la dérivée de ces grandeurs en fonction de la longueur d'onde. La modulation de la longueur d'onde est obtenue par miroir vibrant. La source est constituée par une lampe à iode de $75 \mathrm{~W}$ située à l'intérieur d'une enceinte réfrigérée. Le signal de sortie est mesuré à l'aide d'un photomultiplicateur RT 56 CVP dans la bande spectrale de travail 1,3$2,5 \mathrm{eV}$. Un traitement analogique des signaux permet d'enregistrer directement les différentes grandeurs mesurées. Les détails techniques de cet appareil ont été décrits ailleurs [15].

3.1 COEFFICIENT D'ABSORPTION. - Le coefficient d'absorption $\alpha$ se déduit des mesures de la réflectivité $R(\omega)$, de la transmission $T(\omega)$ à l'aide de la relation :

$\left.\alpha=-\frac{1}{d} \log \left[\left(1-R_{12}\right)^{4}+4 T^{2} R_{12}^{2}\right)^{1 / 2}-\left(1-R_{12}\right)^{2}\right]$

qui tient compte des réflexions multiples à l'intérieur d'une lame d'épaisseur $d$ :

$$
R_{12}=\frac{(n-1)^{2}+\kappa^{2}}{(n+1)^{2}+\kappa^{2}} \simeq \frac{(n-1)^{2}}{(n+1)^{2}}
$$


est le coefficient de réflexion qui serait mesuré sur un échantillon d'épaisseur infinie, exprimée en fonction de l'indice complexe $n+i \kappa$; il ne dépend pratiquement que de l'indice de réfraction.

On a utilisé les valeurs de l'indice de réfraction mesurées par Casey et al. [9] en fonction de la composition. Il peut être considéré comme indépendant de la longueur d'onde dans la bande où se situent nos mesures pour un échantillon donné (une centaine de meV autour de la transition).

L'épaisseur $d$ de l'échantillon a été déduite de la position des pics d'interférence qui apparaissent au-dessous du seuil d'absorption, à l'aide de la relation

$$
d=\frac{\lambda_{1} \lambda_{2}}{2 n\left(\lambda_{1}-\lambda_{2}\right)}
$$

où $\lambda_{1}, \lambda_{2}$ désignent les longueurs d'onde de deux extréma successifs.

La figure 2 représente les résultats obtenus pour trois échantillons de $\mathrm{Ga}_{1-x} \mathrm{Al}_{x} \mathrm{As}$, à $300 \mathrm{~K}$ et $77 \mathrm{~K}$, dont la composition $x$ en aluminium est donnée tableau I.

Le coefficient d'absorption peut être calculé à partir du taux de transition par unité de temps $W(\omega)$ d'un état $\left(v, \mathbf{k}_{\mathbf{v}}\right)$ de la bande de valence vers un état $\left(c, \mathbf{k}_{\mathrm{c}}\right)$ de la bande de conduction, sous l'effet du

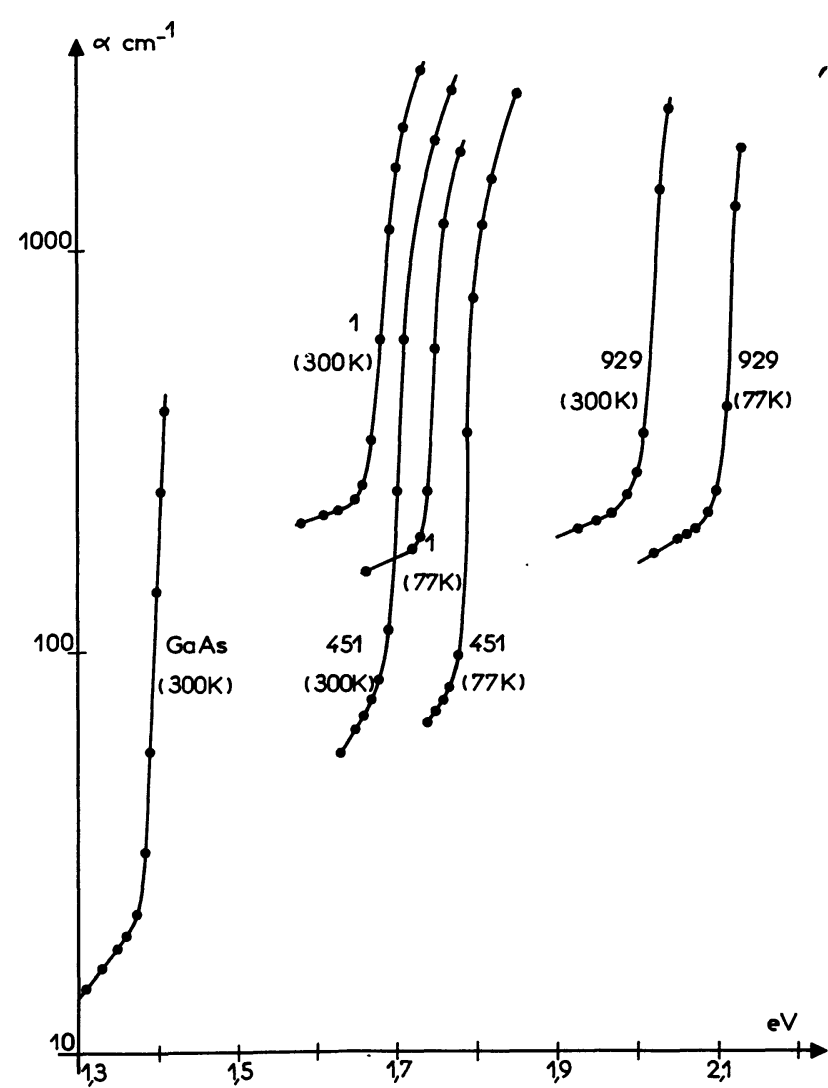

Fig. 2. - Coefficient d'absorption obtenu dans trois échantillons de compositions différentes, au voisinage de la transmission fondamentale $\Gamma_{15}^{\mathrm{v}} \rightarrow \Gamma_{1}^{\mathrm{c}}$.

[Absorption coefficient measured in three samples of different compositions, in the vicinity of the fundamental transition $\Gamma_{15}^{\mathrm{v}} \rightarrow \Gamma_{1}^{\mathrm{c}}$.] champ électromagnétique de fréquence $\omega$, en présence de collision. Compte tenu de la règle de sélection habituelle $\mathbf{k}_{\mathrm{v}} \simeq \mathbf{k}_{\mathrm{c}}=\mathbf{k}, W(\omega)$ peut être mis sous la forme :

$$
W(\omega) \alpha \frac{\Gamma / 2}{\Gamma^{2} / 4+\left[E_{\mathrm{c}}(\mathbf{k})-E_{\mathrm{v}}(\mathbf{k})+\delta E-\hbar \omega\right]^{2}}
$$

$E_{\mathrm{v}}(\mathbf{k}), E_{\mathrm{c}}(\mathbf{k})$ énergie des états électroniques dans chacune des bandes ; $\delta E$ est le déplacement en énergie et $\Gamma$ le paramètre d'élargissement résultant des collisions.

La transition optique a lieu au voisinage de $\mathbf{k}=0$. En supposant les deux bandes paraboliques dans cette région de la zone (point de Van Hove du type $M_{0}$ ), on obtient par sommation sur tous les couples $(v \mathbf{k}, c \mathbf{k})$, le coefficient d'absorption sous la forme :

$$
\begin{aligned}
& \alpha(\omega)=\frac{B}{\hbar \omega} \times \\
& \times \frac{\hbar \Gamma /^{2}}{\left[\left[\left(E_{0}+\delta E-\hbar \omega\right)^{2}+\frac{\hbar^{2} \Gamma^{2}}{4}\right]^{1 / 2}+E_{0}+\delta E-\hbar \omega\right]^{1 / 2}}
\end{aligned}
$$

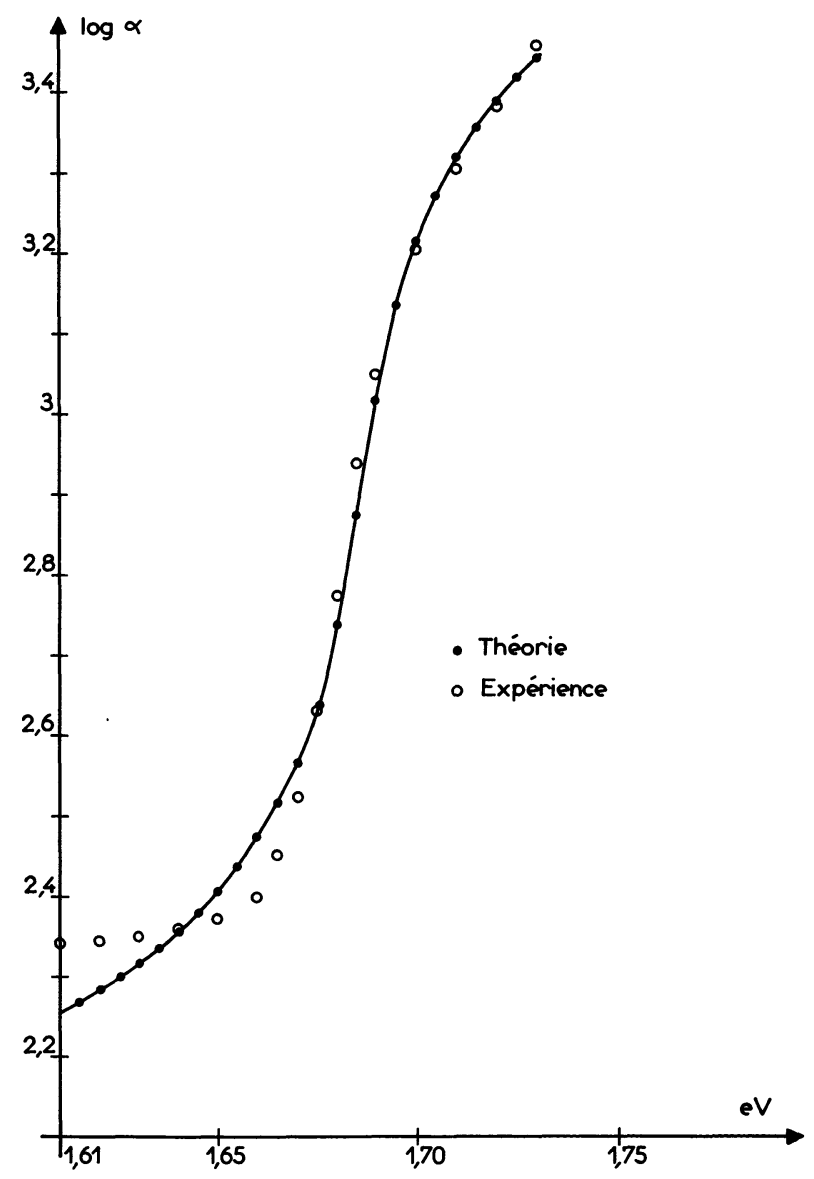

Fig. 3. - Ajustement par méthode des moindres carrés de l'expression théorique du coefficient d'absorption avec les résultats expérimentaux obtenus sur l'échantillon 1 .

[Theoretical curve obtained by making a least-squares fit of the selected experimental points of the sample 1.] 
où $B$ est une constante qui ne dépend de $\omega$ que par l'intermédiaire de l'indice $n$ et $E_{0}$ la largeur de la bande interdite en $\mathbf{k}=0$.

Nous avons ajusté l'expression précédente à la courbe expérimentale par la méthode des moindres

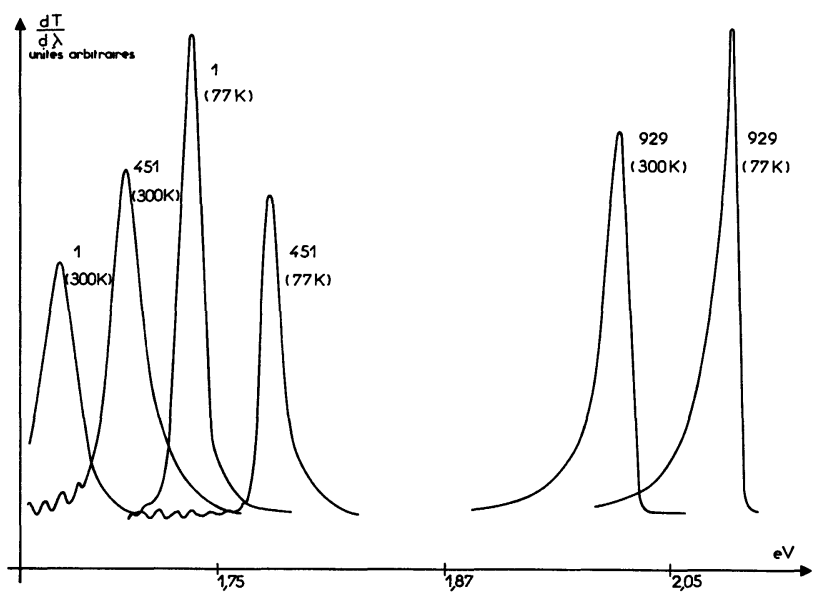

Fig. 4. - Dérivée de la transmission mesurée par modulation de longueur d'onde dans les trois échantillons étudiés.

[Transmission derivative obtained by wavelength modulation in three samples.]

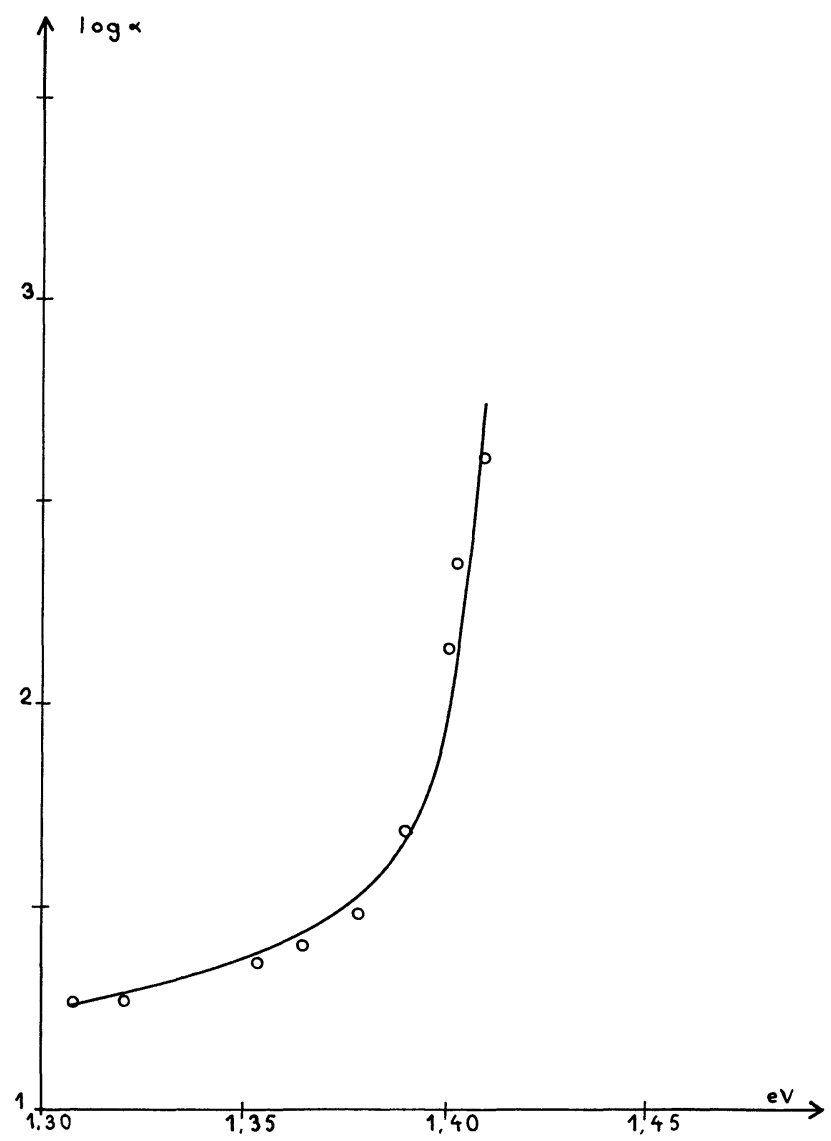

Fig. 5. - Ajustement par méthode des moindres carrés de l'expression théorique du coefficient d'absorption avec les résultats expérimentaux obtenus sur GaAs.

[Theoretical curve obtained by making a least-squares fit of the selected experimental points of GaAs.] carrés. Le résultat est représenté figure 3 pour l'échantillon no 1 , à $300 \mathrm{~K}$.

L'accord est satisfaisant sauf à basse énergie. L'hypothèse de la bande parabolique n'est probablement pas justifiée dans cette région du fait de la dispersion du seuil d'absorption due aux fluctuations de la densité d'impuretés et de la composition. A titre de comparaison nous donnons figure 5 le résultat pour un échantillon de GaAs de type $n$ avec $n=5 \times 10^{15} / \mathrm{cm}^{3}$.

Nous donnons dans le tableau I les valeurs ainsi obtenues de $E_{0}+\delta E$ et $\Gamma$ pour les différents échantillons étudiés, aux températures de $300 \mathrm{~K}$ et $77 \mathrm{~K}$. Le déplacement $\delta E$ est de l'ordre de $\Gamma$. Il peut être calculé si l'on connaît les mécanismes de collision prédominants.

3.2 Transmission moduléE. - Le coefficient de transmission $T(\omega)$ est donné par l'expression :

$$
T(\omega)=\frac{\left(1-R_{12}\right)^{2} \exp (-\alpha d)}{1-R_{12}^{2} \exp (-2 \alpha d)} .
$$

Le dénominateur étant pratiquement égal à 1 $\left(R_{12} \sim 0,32\right.$ et $\left.\alpha d \sim 1\right)$, l'expression (5) s'écrit plus simplement :

$$
T(\omega) \cong\left(1-R_{12}\right)^{2} \exp (-\alpha d) .
$$

Négligeant les variations de $R_{12}$ dans la bande fréquence considérée, la dérivée $T(\omega)$ par rapport à $\omega$ s'écrit

$$
\frac{\mathrm{d} T}{\mathrm{~d} \omega}=-\left(1-R_{12}\right)^{2} \exp (-\alpha d) d \frac{\mathrm{d} \alpha}{\mathrm{d} \omega}
$$

si nous remplaçons $\mathrm{d} \alpha / \mathrm{d} \omega$ par son expression déduite de (4), on trouve que $\mathrm{d} T / \mathrm{d} \omega$ présente un maximum très proche de

$$
\hbar \omega=E_{0}+\delta E .
$$

La mesure du pic de transition dérivée fournit donc très simplement l'énergie de la transition (au déplacement près).

On peut également en déduire le paramètre d'élargissement $\Gamma$ en mesurant la largeur du pic à une hauteur déterminée. Nous avons choisi la largeur à $90 \%$ $\mathrm{du}$ maximum pour que l'exponentielle qui figure en (7) ait sensiblement la même valeur qu'au maximum. On trouve alors

$$
\Delta \hbar \omega(\text { à } 90 \% \text { en } \mathrm{eV})=2,6 \hbar \Gamma / 2 e
$$

où $e$ est la charge de l'électron.

Les pics obtenus sur les trois échantillons étudiés sont représentés figure 4 . Les valeurs de $E_{0}+\delta E$ et du paramètre d'élargissement $\Gamma$ que l'on en tire par la méthode précédemment décrite sont rassemblées dans le tableau I. On constate que les deux méthodes présentent un accord très satisfaisant, compte tenu 
des erreurs expérimentales et des approximations faites pour simplifier les calculs.

Les valeurs de la composition en aluminium $x$ indiquées dans le tableau 1 ont été déduites de nos mesures antérieures [4], en accord d'ailleurs avec celles de Casey et Panish [1].

4. Conclusion. - Nous avons montré qu'à l'aide d'une technique simple, il est possible d'éliminer le substrat en GaAs de couches épitaxiales de $\mathrm{Ga}_{1-x} \mathrm{Al}_{x} \mathrm{As}$ de différentes compositions et de quelques dizaines de $\mu \mathrm{m}$ d'épaisseur.

L'élimination du substrat permet d'effectuer des mesures de transmission et de calculer le coefficient d'absorption dont la connaissance est très importante dans les applications.

La dérivée de la transmission obtenue par spectroscopie à modulation de longueur d'onde fournit une deuxième méthode très commode pour mesurer la position en énergie et la largeur de la transition fondamentale $\Gamma_{15}^{\mathrm{v}} \rightarrow \Gamma_{1}^{\mathrm{c}}$.

Remerciements. - L'auteur remercie la Radiotechnique-Compelec, Centre de Caen, qui a fourni gracieusement les couches épitaxiales de GaAlAs étudiées dans cet article.

\section{Bibliographie}

[1] Casey, H. C. Jr and Panish, M. B., J. Appl. Phys. 40 (1969) 4910.

[2] Shah, J., Miller, B. I. and Digiovanni, A. E., J. Appl. Phys. 43 (1972) 3436.

[3] Bumelis, A. I., Maltudis, E. K., Matulenis, A. Yu., ShimKyavichus, Ch. I. and Shimulite, E. A., Fiz. Tekh. Poluprovodn. 9 (1975) 1636, Sov. Phys. Semicond. 9 (1976) 1081

[4] Lande, R., Madelon, R., Hairie, A. et Fortini, A., Revue Phys. Appl. 12 (1977) 483.

[5] Rogulin, V. Yu. and Shlenskin, A. A., Fiz. Tekh. Poluprovodn 7 (1978) 988 ; Sov. Phys. Semicond. 7 (1973) 673.

[6] Monemar, B., Shih, K. K. and Petit, G. D., J. Appl. Phys. 47 (1976) 2604

[7] Berolo, O. and Woolley, J. C., Can. J. Phys. 40 (1969) 4910.
[8] Pollak, F. H., OKeke, C. E., Vanier, P. E. and Raccah, P. M J. Appl. Phys. 49 (1978) 4216.

[9] Casey, H. C. Jr, Sell, D. D. and Panish, M. B., Appl. Phys. Lett. 24 (1974) 63.

[10] Sommers, H. S. Jr and Cockwood, H. F., J. Appl. Phys. 48 (1977) 4000.

[11] Haga, E. and Kimura, H., J. Phys. Soc. of Japan 19 (1964).

[12] Hilsun, C., Semicond. and Semimetal. (Acad. Press) (1966) 3.

[13] Onton, A., 10th Int. Conf. on Phys. of Semicond. (1970) p. 107.

[14] Hess, E., Topol. I., Schulze. K. R., Neumann, H. and L VGer. K.. Phis. Status Solld 55 (1973) A 87.

[15] Logan, R. A. and Reinhart, F. K., J. Appl. Phys. 44 (1975) 4172.

[16] Rapport de fin de contrat D.G.R.S.T. (1976) $\mathrm{n}^{\circ}$ 173-7-1168. 